\title{
Understanding user Emotions Through Interaction with Persuasive Technology
}

\author{
Wan Nooraishya Wan Ahmad ${ }^{1}$ \\ Ahmad Rizal Ahmad Rodzuan ${ }^{3}$ \\ Faculty of Computing and Informatics \\ Universiti Malaysia Sabah, Labuan International Campus \\ F.T. Labuan, Malaysia
}

\author{
Nazlena Mohamad $\mathrm{Ali}^{2}$ \\ Institute of Industrial Revolution 4.0 (IIR4.0) \\ Universiti Kebangsaan Malaysia \\ Bangi, Selangor. Malaysia
}

\begin{abstract}
Emotions play a vital role in persuasion; thus, the use of persuasive applications should affect and appeal to the users' emotions. However, studies in persuasive technology have yet to discover what triggered the users' emotions. Therefore, the objectives of this study are to examine user emotions and to identify the factors that affect user emotions in using persuasive applications. This study is conducted in three stages; preinteraction, during-interaction and post-interaction, employed a mixed-method approach using Geneva Emotions Wheel (GEW) and open-ended survey questions that analyzed using thematic analysis. The result shows that most of the emotions that users felt belong to high-control positive valence emotions that consist of interest, joy and pleasure. User, system and interaction are the three factors that triggered the emotions encompasses of elements such as Individual Awareness, Personality, Interface Design, Persuasive Function, Content Presentation, System Quality, Usability, and Tasks. The findings contribute to the body knowledge of Persuasive Technology, where the discovered factors and its elements are the antecedents that should be the concern in constructing an emotion-based trust design framework that could bring emotional impact to users to ensure a successful persuasion.
\end{abstract}

Keywords-Emotion; emotional states; interaction; persuasive technology; captology

\section{INTRODUCTION}

Persuasive technology (PT) is increasingly being developed commercially and has been one of the first research area related to shaping human behavior. It is a technology with a purpose to shape and/or change people's attitudes or behavior towards an issue [1]. For example, a persuasive application is developed to help and assist a smoker in becoming a non-smoking person. The persuasion process is to be done without using coercion but using many strategies such as social influence, self-monitoring, and personalization that capable of triggering emotions in users [2]. The PT work is often concerned with the use of practical approaches to address particular behavioral problems [3], hence making the emotional effect that one feels while using PT has been overlooked. Since the persuasion strategies are capable of triggering emotions, it is essential to know what exactly the users feel when using persuasive technology and what makes them feel those emotions. Persuasive technology must bring an emotional impact to the users to ensure the success of the persuasion process.
Recently, studies on emotion have become increasingly important as the need to incorporate emotion into computer application design has become a significant focus of $\mathrm{HCI}$ (Park, Lee, \& Kim, 2011). However, most studies on emotion in the field of HCI focus more on the emotional stimulation of users during interactions [4][5][6], although studies on emotion can be done at all levels of interactions between computers and humans [7]. Thus, studies on emotions involving all levels of interaction between humans and computers have yet to be discovered.

Hence, the objectives of this study are 1) to investigate the emotions of the user at three interaction stages; pre, during and post, and 2) to identify the factors that trigger user emotions in using persuasive technology. The next section of this paper will further describe the emotion and how it contributes to persuasion as well as persuasive technology. The methodology section explains the comprehensive methods used for data collection, while the data analysis section elaborates on the methods used to analyze the collected data. Next, the result and discussion are presented to explain the discovered scenario. Lastly, the paper ends with a conclusion.

\section{LITERATURE REVIEW}

Persuasion involves an attempt to bring about a change in attitudes or behaviors as a result of providing information on a topic or issue without intimidation. Significantly, attitudes are not only relying on thoughts and beliefs but also feelings and emotions [8]. Emotions are one of the components that influence the user experience, which is a medium for users to understand how they feel about using a technology [9]. It is defined as a physiological state of arousal that is triggered by beliefs about something which consists of cognitive physiological, social and behavioral aspects [10]. Emotions are sensitive and are stimulated by situations due to certain circumstances, actions or objects last for a short period [11]. This distinguishes between emotion and mood because the mood lasts for an extended period, which can last for weeks or months, occurring without a specific target and is often separate from what causes the mood to be triggered [12]. According to [13], emotions are stimulated by the culture in which they are produced through actions or tasks and interactions.

This study is sponsored by the Fundamental Research Grant Scheme (FRGS) (FRGS/2/2014/ICT01/UKM/02/3) by the Ministry of Education. 
Emotions can be categorized in a variety of ways. Discrete emotions, often associated with facial expressions, are described as basic emotions. A study conducted by [14] showed that each of the people recognizes and expresses basic emotions in much the same way as others. There are currently six basic emotions that are universally expressed and recognized throughout the region and culture, which are happy, angry, sad, scared, disgusted and shocked [15]. Dimensional emotions are an exciting category of emotions because they offer a way to describe and distinguish emotional states [16]. Compared to discrete emotions that have only a few emotions, dimensional emotions are subdivided into bipolar dimensions of pleasure-displeasure, arousal, and dominance-submissiveness. Dimensional emotion assesses human feelings in terms of valence from positive to negative and arousal [11]. Measurement of the dimension of arousal from active to passive explains whether or not human actions are affected by emotional states. Dimensional emotions are better able to deal with non-discrete emotions as well as variations in an emotional state over time. For example, the angry feelings are considered to be negative valence with high arousal. At the same time, sadness is associated with negative valence with low arousal, indicating that the same category of emotional valence will have different levels of arousal. In this study, the emotional state's data refers to the stimulus data resulting from the use of persuasive technology that is likely to consist of one or more combinations of emotions.

Emotions can then be modelled as a form of information processing and another set of inputs into cognitive processes [13] where emotion can be a form of internal signal that contributes to cognitive actions to change one's attitude and behaviour. Individuals with positive emotions are more likely to be motivated to change their behaviours [17]. The research showed that emotions and website design influence a person's behavioural shift as a website that uses cue representation to view information is beneficial for individuals with positive emotions. Thus, [18] believes that understanding user emotions is one of the critical components in creating an impactful application apart from putting trust and persuasion in the design. He emphasized that simply providing persuasion technology might at first be useful, but over time, it created a "mess of persuasion", reduced trust and was no longer sufficient. Therefore, designers need to develop persuasive tools based on a deep understanding of the user's emotions.

Thus, the above-reviewed papers show that there is a gap in understanding user's emotions, especially in the interaction with persuasive technology. It is important to know precisely what makes the user feel a particular emotion when using persuasive technology because this might become the antecedents that could bring emotional impact to improve the persuasion process.

\section{RESEARCH Methodology}

The methodology process consists of five parts: (a) participants, (b) material, (c) study design, (d) measures, and (e) setting and task. We present each part as a subsection here.

\section{A. Participants}

The participants were recruited through advertisements via Facebook. Due to the use of persuasive health applications in the study, such participants have undergone an evaluation using a questionnaire [19] to evaluate their readiness to improve the state of performing physical activity. The volunteers are qualified for the study if only they are in the state of contemplation, preparation, action, and maintenance based on the assessment results. These results not only showed the readiness level of behavior change towards physical activities but also as an indicator of committing to the study. The study was conducted in 6 weeks, with 25 participants managed to complete all three stages of interaction. They consist of 10 males and 15 females. The participants were among the university students and employees. Among the participants, 18 of them had experience in using similar persuasive applications with less than six months and between 6 months to a year experience. The participants' age was in the range from 21 to 45 years old.

\section{B. Material}

The process of determining appropriate persuasive applications to be studied, ranging from online searching, screening and exclusion. A list of persuasive applications regarding health and environmental was established from Google search activity using different keywords. For example, keywords such as "top health applications in Malaysia" and "top health apps" are used to find persuasive applications on health; meanwhile, keywords such as "game for change" and "persuasive games" are used to search for persuasive applications on the environment.

Three levels of criteria with overall 11 operational variables from [20] were used to screen the 63 persuasive applications gathered from the Internet. The first level of screening is to find applications that fulfil the needed definition of a persuasive application using two operational variables, which are persuasive application and app platform. The second level of screening consists of seven operational variables that were used to find applications that fulfil the specific criteria of a needed persuasive application. The seven operational variables are the theme, type of app, target user, delivery, app availability, interactivity styles, and device collaboration. The third screening level is to ensure that the content of the listed persuasive applications is fit to all range of users regardless of country and education background and meet the local community demands. Two operational variables that were used in the third screening process are app content and the focus of the content.

The exclusion process runs simultaneously with the screening process, where finally, five persuasive applications are selected for this study. All five applications have been identified to qualify as persuasive technology, as mentioned by [1] and [2]. Three from the five applications are about health, and another two applications are on the environment. Both health and environment applications fall under different categories of persuasive applications. Health applications that consist of MyFitnessPal, MapMyFitness and Fitocracy are in tool category that shared the same goal to support and enhance user capabilities to achieve the desired target behavior. 
Meanwhile, the selected environment applications fall under medium category shared a goal to showcase the relationship of cause and effect thru simulation consist of Stop Disaster and Pandemic 2 game. Table I describes all five persuasive applications.

\section{Study Design}

A mix of between-subject and within-subject design is used to design participants for the experiment. Each participant has the opportunity to use both types of persuasive applications simultaneously within six weeks. Participants were assigned to six groups randomly so that each participant in a group can use a pair of different applications. However, the groups were limited to several participants at a time. Group 1 participants were given a pair of MyFitnessPal and Stop Disaster. Group 2 used MyFitnessPal and Pandemic 2, while participants in group 3 get to used MapMyFitness and Stop Disaster. Meanwhile, participants in group 4 get to used MapMyFitness and Pandemic 2, while group 5 participants get a chance to use Fitocracy and Stop Disaster. Group 6 participants were given a pair of Fitocracy and Pandemic 2.

\section{Measures}

A questionnaire that consists of ratings and open-ended questions related to emotional states construct is used as the measurement instrument. For each interaction stage, participants were asked to pick five emotional states to construct which they felt and rated the intensity of the emotions as well as stating the cause that makes them felt the emotions. Geneva Emotion Wheel (GEW) [11] [21] was used to measure emotional states construct. GEW is chosen since it has been used in various fields to study the user's emotions related product and technology. Studies [22] and [23] employed GEW to study emotions towards virtual learning environments. A study by [24] evaluates user emotions related to coffee machines and alarm clock, while in [25] the GEW is used to assess user emotions towards a higher learning website.

TABLE I. PERSUASIVE APPLICATIONS

\begin{tabular}{|l|l|l|}
\hline Type & Application & Description \\
\hline \multirow{4}{*}{ Tool } & MyFitnessPal & $\begin{array}{l}\text { A tracking tool to help weight loss and remain } \\
\text { physically fit based on calorie food intake and } \\
\text { exercise. }\end{array}$ \\
\cline { 2 - 3 } & MapMyFitness & $\begin{array}{l}\text { A tracking tool to record food intake and } \\
\text { exercises by calculating intake and burned } \\
\text { calories. }\end{array}$ \\
\cline { 2 - 3 } & Fitocracy & $\begin{array}{l}\text { A social network for fitness that tracks users' } \\
\text { fitness progress using gamification principles } \\
\text { such as the quest, points and level up to } \\
\text { encourage people. }\end{array}$ \\
\hline Stop Disaster & $\begin{array}{l}\text { A simulation game on the prevention of natural } \\
\text { disasters. The game educates users in } \\
\text { preventing natural disasters from getting worse } \\
\text { and reducing the cost of destruction from the } \\
\text { disaster. }\end{array}$ \\
\cline { 2 - 3 } & Pandemic 2 & $\begin{array}{l}\text { A simulation game on disease spreading. The } \\
\text { game teaches users how diseases spread } \\
\text { throughout the world. }\end{array}$ \\
\hline
\end{tabular}

GEW composed of 20 discrete emotions arranged parallel in the form of circles according to emotional groups divided into two dimensions of valence (positive and negative) and control (high-low). The division of valence and control dimensions have split the 20 discrete emotions into four groups; positive valence-high control (i.e., interested, amusement, pride, joy, pleasure), positive valence-low control (i.e., contentment, love, admiration, relief, compassion), negative valence-high control (i.e., anger, hate, contempt, disgust, fear), and negative valence-low control (i.e., disappointment, shame, regret, guilt, sadness). The emotional states construct was measured using a 5-point Likert scale, illustrates the intensity of the emotions from low intensity (towards the wheel center) to high intensity (towards the wheel circumference). Five out of 20 emotions from the GEW that were rated by users were listed as the most frequent or dominant emotions that users felt. For each five listed emotions, participants are required to outline the cause that makes them felt those emotional responses.

\section{E. Setting and Tasks}

For this study, user emotional states and also the reasons that triggered the emotions were studied in three interaction stages; pre-interaction, during-interaction, and postinteraction. The pre-interaction stage is defined as an initial interaction with the persuasive applications that they are assigned. During-interaction is a stage where participants used the persuasive applications on their own without the help of others according to their needs and free time. Post-interaction is a stage where the participant has passed a specific period in using the persuasive applications.

The same set of questionnaires each to assess tool and medium type of persuasive applications were distributed in the lab at a specific seat. Upon arrival, participants were asked to fill up the demographic details in the questionnaire. Each interaction stages consist of two sessions of experiment and one relaxing session at the beginning of every session using Calm - a web application that provides scenic pictures and the soothing sound of nature to eliminate stress and stabilizing emotions. When participants tested different applications (AP1: tool, AP-2: medium), the same experimental procedure was repeated. The following description describes further details of each procedure according to the interaction stage:

- Pre-interaction: It takes about 1 hour to complete two sessions of the experiment. Participants spend 5 minutes for relaxing as a form of control to emotions at the beginning of each session using Calm web application. Before the participants were permitted to have initial interaction with the applications, a demo on how to use the applications was showed to them using slide presentation. In the first session of the experiment that takes about 20 minutes, participants get to explore the AP-1 app based on the tasks given include answering a questionnaire after finishing the tasks. In the next twenty minutes, the participants are required to explore the AP-2 app based on the tasks give and answer a questionnaire after the tasks completed. 
- During-interaction: Before the first session of the experiment begun, participants rested for 5 minutes by wearing a headphone to use the Calm web application. With about 20 minutes for each session, participants are required to perform a list of tasks using the AP-1 app and another 20 minutes using the AP-2 app. After the tasks completed, the participants are then required to answer the questionnaires provided, referring to the application they used.

- Post-interaction: The study at this stage was conducted in week six, where the lab experiment is no longer necessary. In the two previous stages of the experiment, participants spent 5 minutes' rest at the beginning of each session for emotional relaxation. Afterwards, participants need to answer a provided questionnaire each to evaluate the post-use of AP-1 and AP-2 apps. The participants received a token of appreciation after completed both questionnaires.

In general, participants are required to perform the tasks for AP-1 and AP-2 applications with a minimum addition of new tasks based on the interaction stage. Below are the given tasks:

- AP-1: For the pre-interaction stage, participants must first register a user account using either an email or Facebook for the health application (i.e., MyFitnessPal, MapMyFitness, Fitocracy) to which they have been assigned before are allowed setting up a user profile and target goal. Next, participants created physical and/or nutritional activities daily by using the available databases. To start with, participants must record diaries "yesterday" and "today." Finally, participants must see the feedback or advice provided based on the data entered. For the during-interaction stage, participants are generally required to perform the same tasks as the pre-interaction stage. Additional tasks have been established where participants are expected to assess specific functions in AP-1 applications, such as the Body Mass Index (BMI), Body Metabolic Rate (BMR), and other actions that can be taken to obtain feedback or progress reports from the system.

- AP-2: In the pre-interaction stage, first, for the Stop Disaster game, participants are required to choose the "easy" game level. For Pandemic 2, participants are required to choose the "virus" role play from the role options such as bacteria and parasites. Within the specified time and/or budget, participants played the game to accomplish a mission. Finally, participants viewed their achievements from the report provided by the system. In the during-interaction stage, for the Stop Disaster game, participants are required to choose the "hard" game level. For the Pandemic 2 game, participants are required to choose the "parasites" role play. Finally, participants are required to complete the game's mission and see their achievements through a report provided by the system.

\section{DATA ANALYSIS}

The combination of quantitative and qualitative analysis is used to analyze the data from the questionnaire. Using the SPSS software to conduct the quantitative analysis, descriptive analysis was used to analyze user emotional states in three interaction stages as well as the demographic data. Frequency values from the descriptive analysis are used to determine the frequency of users' triggered emotions for each stage of interaction for both persuasive applications. One-way repeated measure ANOVA is used to study the changes in emotional state intensity towards persuasive applications at different interaction stages. This analysis is also used to investigate the changes in user emotions towards persuasive technology as a whole over time. A significant value benchmarked less than .05 is used in determining the statistical significance of the analysis.

For qualitative data, inductive content-analysis coding [26] is used to identify factors that stimulate user emotions when using persuasive technology. It composes four phases; data preparation, data coding, categorization, and theme analysis. In the data preparation phase, questionnaire transcripts for the three interaction stages are arranged by the types of persuasive applications to ensure that the coding process can be distinguished before the general set is generated by merging the existing code. In the coding phase, transcripts text is check and labelled appropriately to create open codes. The inductive analysis method is used in the categorization phase to construct categories based on the obtained open codes. The theme analysis phase performs an overall analysis of the constructed categories to form a theme that shared the same pattern of characteristics.

\section{FINDINGS}

We present the findings into two parts: a) emotional states and b) stimulation factors of emotions to answer the two research objectives.

\section{A. Emotional States}

Fig. 1 indicates the frequency of user emotions stimulated from the pre-interaction stage for both persuasive applications. From the first impression, the persuasive application AP-1 often arouses emotions such as "interest", "pleasure", "love", "joy", "admiration," and also "disappointment" in the user. Those emotions consist of high-control positive emotions (interest, pleasure and joy), low-control positive emotion (admiration) and also low-control negative emotions (disappointment). Meanwhile, emotions such as "interest", "amusement", "pleasure", "contentment," and "disappointment" dominate the user emotions at first encounter towards persuasive applications AP-2. Table II summarizes the frequency of user emotions for the preinteraction stage. High-control positive emotions dominate the emotions aroused by AP-1 at the user's first encounter compared to AP-2, where user emotions were dominated by a mixture of both positive and negative emotions but dominated slightly more by high-control positive emotions compared to low-control negative emotions. 


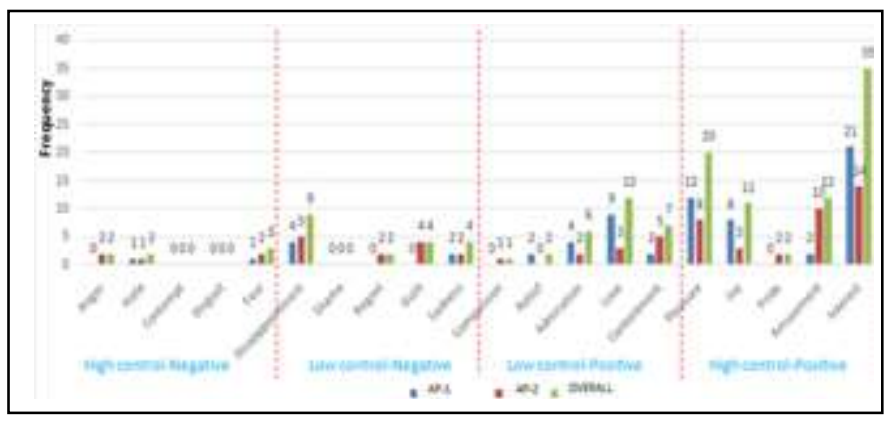

Fig. 1. Frequencies of user Emotions in the Pre-Interaction Stage.

TABLE II. FREQUENCY OF USER EMOTIONS ACCORDING TO GROUPS OF EMOTIONS FOR THE PRE-INTERACTION STAGE

\begin{tabular}{|l|l|l|l|l|l|l|}
\hline \multirow{2}{*}{$\begin{array}{l}\text { Emotion } \\
\text { Groups }\end{array}$} & \multicolumn{3}{|c|}{ Emotion Frequency } & \multicolumn{3}{c|}{ Emotion Intensity Mean } \\
\cline { 2 - 7 } & $\boldsymbol{A P}-\mathbf{1}$ & $\boldsymbol{A P}-\mathbf{2}$ & $\boldsymbol{A L L}$ & $\boldsymbol{A P}-\mathbf{1}$ & $\boldsymbol{A P}-\mathbf{2}$ & $\boldsymbol{A L L}$ \\
\hline $\begin{array}{l}\text { High control - } \\
\begin{array}{l}\text { Negative } \\
\text { Valence }\end{array}\end{array}$ & 2 & 5 & 7 & 2.00 & 3.33 & 3.17 \\
\hline $\begin{array}{l}\text { Low control - } \\
\text { Negative } \\
\text { Valence }\end{array}$ & 6 & 13 & 19 & 4.13 & 3.76 & 3.88 \\
\hline $\begin{array}{l}\text { Low control - } \\
\text { Positive } \\
\text { Valence }\end{array}$ & 17 & 11 & 28 & 3.66 & 4.38 & 3.86 \\
\hline $\begin{array}{l}\text { High control - } \\
\text { Positive } \\
\text { Valence }\end{array}$ & 43 & 37 & 80 & 4.02 & 4.42 & 4.30 \\
\hline
\end{tabular}

Fig. 2 shows the frequency of user emotions in a duringinteraction stage. Using AP-1, users often aroused with "interest", "contentment", "love", "relief," and "disappointment" emotions. These emotions consist of highcontrol positive emotions (interest), low-control positive emotions (contentment, love and relief) and also low-control negative emotions (disappointment). Meanwhile, the use of AP-2 often stimulated users feeling with emotions of "interest", "relief", "sadness", "disappointment," and some "amusement", "joy," and "pleasure". All the emotions that the users felt consisted of 3 emotional groups: high-control positive emotions (interest, amusement, joy and pleasure), low-control positive emotions (relief) and low-control negative emotions (sadness, disappointment). Table III summarizes the frequency of user emotions at the duringinteraction stage. Positive emotional groups dominate the emotions aroused by both AP-1 and AP-2 with high control.

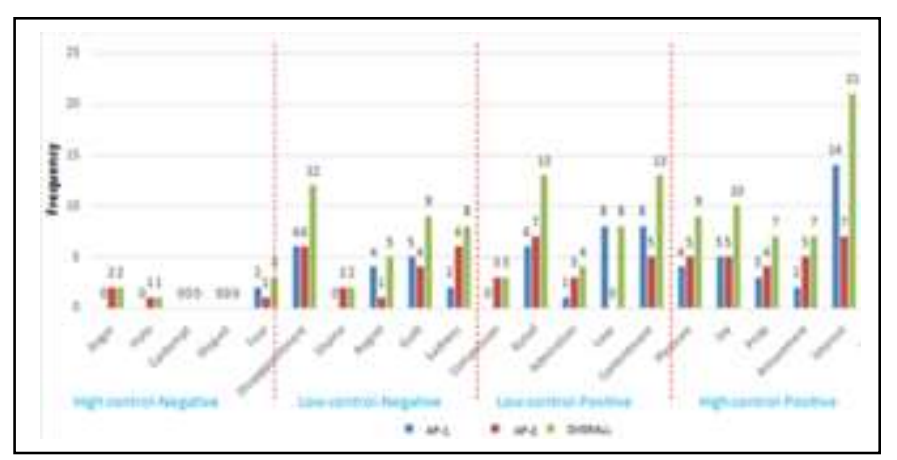

Fig. 2. Frequencies of user Emotions in the During-Interaction Stage.
TABLE III. FREQUENCY OF USER EMOTIONS ACCORDING TO GROUPS OF EMOTIONS FOR DURING-INTERACTION STAGE

\begin{tabular}{|l|l|l|l|l|l|l|}
\hline \multirow{2}{*}{$\begin{array}{l}\text { Emotion } \\
\text { Groups }\end{array}$} & \multicolumn{3}{|c|}{ Emotion Frequency } & \multicolumn{3}{c|}{ Emotion Intensity Mean } \\
\cline { 2 - 7 } & $\boldsymbol{A P}-\mathbf{1}$ & $\boldsymbol{A P}-\mathbf{2}$ & $\boldsymbol{A L} \boldsymbol{L}$ & $\boldsymbol{A P}-\mathbf{1}$ & $\boldsymbol{A P}-\mathbf{2}$ & $\boldsymbol{A L L}$ \\
\hline $\begin{array}{l}\text { High control - } \\
\begin{array}{l}\text { Negative } \\
\text { Valence }\end{array}\end{array}$ & 2 & 4 & 6 & 2.50 & 4.00 & 3.89 \\
\hline $\begin{array}{l}\text { Low control - } \\
\text { Negative } \\
\text { Valence }\end{array}$ & 17 & 8 & 25 & 3.41 & 3.75 & 3.66 \\
\hline $\begin{array}{l}\text { Low control - } \\
\text { Positive } \\
\text { Valence }\end{array}$ & 23 & 9 & 32 & 3.86 & 3.75 & 3.85 \\
\hline $\begin{array}{l}\text { High control - } \\
\text { Positive } \\
\text { Valence }\end{array}$ & 28 & 10 & 38 & 3.90 & 4.05 & 3.95 \\
\hline
\end{tabular}

Fig. 3 indicates the frequency of user emotions aroused in the post-interaction stage after six weeks' usage of both persuasive applications. For AP-1, the aroused emotions consist of the feeling of "interest", "love", "pleasure", "contentment", and "disappointment". All of these emotions consist of two groups of positive emotions with high-control (interest, pleasure) and low-control (love, contentment) and also low-control negative emotions (disappointment). For AP2, user emotions often aroused with the feeling of "interest", "amusement", "hate", "disappointment", "pleasure", and "admiration". Table IV summarizes the frequency of user emotions for the post-interaction stage. The analysis shows that user emotions in using AP-1 are more dominated by positive emotions, but are more often dominated by highcontrol positive emotions than low-control emotions. Compared to AP-1, users' emotions towards AP-2 are dominated by low-control emotions but are dominated less by positive emotions than negative emotions.

Additionally, one-way repeated measure ANOVA is conducted to identify the changes in the intensity of user emotional states towards the persuasive applications at Time 1 (pre-interaction), Time 2 (during-interaction) and Time 3 (post-interaction). The mean values and standard deviation are presented in Table V. Result of the analysis shows no statistically significant effect towards time (interaction stages), Wilk's Lambda $=0.93, \mathrm{~F}(2,43)=1.53, \mathrm{p}>0.1$. The result indicates that different interaction stages bring no impact on the intensity of user emotional states.

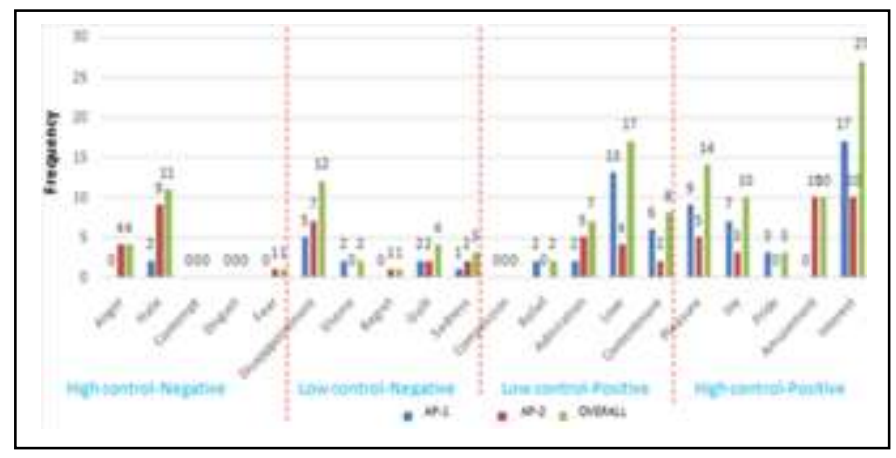

Fig. 3. Frequencies of user Emotions in the Post-Interaction Stage. 
TABLE IV. FREQUENCY OF USER EMOTIONS ACCORDING TO GROUPS OF EMOTIONS FOR POST-INTERACTION STAGE

\begin{tabular}{|l|l|l|l|l|l|l|}
\hline \multirow{2}{*}{$\begin{array}{l}\text { Emotion } \\
\text { Groups }\end{array}$} & \multicolumn{3}{|c|}{ Emotion Frequency } & \multicolumn{3}{c|}{ Emotion Intensity Mean } \\
\cline { 2 - 7 } & $\boldsymbol{A P}-\mathbf{1}$ & $\boldsymbol{A P}-\mathbf{2}$ & $\boldsymbol{A L L}$ & $\boldsymbol{A P}-\mathbf{1}$ & $\boldsymbol{A P}-\mathbf{2}$ & $\boldsymbol{A L L}$ \\
\hline $\begin{array}{l}\text { High control - } \\
\text { Negative } \\
\text { Valence }\end{array}$ & 2 & 14 & 16 & 5.00 & 4.14 & 4.17 \\
\hline $\begin{array}{l}\text { Low control - } \\
\text { Negative } \\
\text { Valence }\end{array}$ & 10 & 17 & 27 & 2.40 & 3.96 & 3.48 \\
\hline $\begin{array}{l}\text { Low control - } \\
\text { Positive } \\
\text { Valence }\end{array}$ & 23 & 18 & 31 & 4.15 & 4.32 & 4.14 \\
\hline $\begin{array}{l}\text { High control - } \\
\text { Positive } \\
\text { Valence }\end{array}$ & 36 & 9 & 45 & 4.04 & 3.59 & 3.90 \\
\hline
\end{tabular}

TABLE V. EMOTIONS DESCRIPTIVE STATISTIC ON THE INTENSITY OF USER EMOTIONAL STATES TOWARDS THE USED OF PERSUASIVE APPLICATIONS AT EACH INTERACTION STAGES

\begin{tabular}{|l|l|l|l|}
\hline Time frame & N & Mean & Std. Dev. \\
\hline Time 1 (pre-interaction) & 45 & 4.10 & 0.80 \\
\hline Time 2 (during-interaction) & 45 & 3.86 & 0.94 \\
\hline Time 3 (post-interaction) & 45 & 3.92 & 1.05 \\
\hline
\end{tabular}

However, the changes in user emotions as a whole towards the persuasive applications at different stages of interaction; Time 1 (pre-interaction), Time 2 (during-interaction) and Time 3 (post-interaction) show a different result. Table VI indicates the mean and standard deviation values for each interaction stage. There is a statistically significant effect towards interaction stages, where Wilk's Lambda $=0.48, \mathrm{~F}$ $(2,47)=25.77, \mathrm{p}<0.001$. The result proved that user emotions changed over time. Nevertheless, the mean value of Table VI explained that even users experienced different emotions at various stages of interaction; the variations in the strength of these emotions were not apparent. Three paired samples t-test were conducted to make post hoc comparisons between the stages. A first paired samples t-test indicated that there was a significant difference between user emotions in pre $(\mathrm{M}=3.00, \mathrm{SD}=0.70)$ and during $(\mathrm{M}=3.82, \mathrm{SD}=0.93)$ interaction stages; $\mathrm{t}(48)=5.83, \mathrm{p}=0.00$. A second paired samples t-test indicated that there was a significant difference between user emotions during $(\mathrm{M}=3.82, \mathrm{SD}=0.93)$ and post $(\mathrm{M}=3.00, \mathrm{SD}=0.79)$ interaction stages; $\mathrm{t}(48)=6.86, \mathrm{p}=0.00$. However, a third paired samples t-test indicated that there was no significant difference between user emotions in pre $(\mathrm{M}=2.97, \mathrm{SD}=0.71)$ and post $(\mathrm{M}=3.02, \mathrm{SD}=0.79)$ interaction stages; $\mathrm{t}(49)=0.34, \mathrm{p}=0.74$.

TABLE VI. DESCRIPTIVE STATISTIC OF EMOTIONAL CHANGES TOWARDS THE USED OF PERSUASIVE APPLICATIONS

\begin{tabular}{|l|l|l|l|}
\hline Time frame & N & Mean & Std. Dev. \\
\hline Time 1 (pre-interaction) & 49 & 3.00 & 0.70 \\
\hline Time 2 (during-interaction) & 49 & 3.82 & 0.93 \\
\hline Time 3 (post-interaction) & 49 & 3.00 & 0.79 \\
\hline
\end{tabular}

\section{B. Stimulation Factors of Emotions}

Table VII shows the coding and categorization for each interaction stage. In total, eight categories have been identified that stimulates emotional states: Individual Awareness, Personality, Interface Design, Persuasive Function, Content Presentation, System Quality, Usability and Task. All eight categories were grouped according to the same characteristics based on the factors of assessment of emotional control by [27] and [28] where three factors were identified: User control, System control and Interaction control. User Control is a users' assessment of their emotions on the basis related to themselves. System Control referred to user emotions that were evoked by the persuasive applications itself- particularly the features used to deliver the persuasion process, while Interaction Control referred to user emotions that were evoked by the interaction between the user and the persuasive applications.

User Control factors consist of two categories: Individual Awareness and Personality. Individual Awareness refers to self-assessment that causes the individual to be aware of what is happening to them, and the individual is aware that he or she is experiencing an event, exhibiting behaviour or having unique characteristics [29]. Individual Awareness constitutes aspects of Consciousness Value and Self-Satisfaction. The definition of the two aspects are as follows:

TABLE VII. CODING AND CATEGORIZATION OF EMOTIONS STIMULATION FACTORS FOR EACH INTERACTION STAGES

\begin{tabular}{|c|c|c|c|c|}
\hline \multirow{2}{*}{ Theme } & \multirow{2}{*}{ Category } & \multicolumn{3}{|c|}{ Open-Code } \\
\hline & & Pre & During & Post \\
\hline \multirow{3}{*}{$\begin{array}{l}\text { User } \\
\text { control }\end{array}$} & \multirow{2}{*}{$\begin{array}{l}\text { Individual } \\
\text { Awareness }\end{array}$} & \multirow{2}{*}{$\begin{array}{l}\text { Self- } \\
\text { satisfaction }\end{array}$} & $\begin{array}{l}\text { Self- } \\
\text { satisfaction }\end{array}$ & $\begin{array}{l}\text { Self- } \\
\text { satisfaction }\end{array}$ \\
\hline & & & $\begin{array}{l}\text { Consciousnes } \\
\mathrm{s} \text { value }\end{array}$ & $\begin{array}{l}\text { Consciousnes } \\
\mathrm{s} \text { value }\end{array}$ \\
\hline & Personality & $\begin{array}{l}\text { Interest } \\
\text { Knowledge } \\
\text { Skill } \\
\text { Relatedness }\end{array}$ & $\begin{array}{l}\text { Interest } \\
\text { Knowledge } \\
\text { Skill } \\
\text { Relatedness }\end{array}$ & $\begin{array}{l}\text { Interest } \\
\text { Knowledge } \\
\text { Skill } \\
\text { Relatedness }\end{array}$ \\
\hline \multirow{10}{*}{$\begin{array}{l}\text { System } \\
\text { control }\end{array}$} & \multirow[t]{2}{*}{$\begin{array}{l}\text { Interface } \\
\text { design }\end{array}$} & $\begin{array}{l}\text { Interface } \\
\text { attractivenes } \\
\text { s }\end{array}$ & $\begin{array}{l}\text { Interface } \\
\text { attractiveness }\end{array}$ & $\begin{array}{l}\text { Interface } \\
\text { attractiveness }\end{array}$ \\
\hline & & Layout & Layout & Layout \\
\hline & $\begin{array}{l}\text { Persuasive } \\
\text { function }\end{array}$ & $\begin{array}{l}\text { Persuasive } \\
\text { function }\end{array}$ & $\begin{array}{l}\text { Persuasive } \\
\text { function }\end{array}$ & $\begin{array}{l}\text { Persuasive } \\
\text { function }\end{array}$ \\
\hline & \multirow{3}{*}{$\begin{array}{l}\text { Content } \\
\text { presentatio } \\
\mathrm{n}\end{array}$} & \multirow{3}{*}{$\begin{array}{l}\text { Information } \\
\text { quality }\end{array}$} & $\begin{array}{l}\text { Information } \\
\text { quality }\end{array}$ & $\begin{array}{l}\text { Information } \\
\text { quality }\end{array}$ \\
\hline & & & $\begin{array}{l}\text { Data } \\
\text { representation }\end{array}$ & $\begin{array}{l}\text { Data } \\
\text { representation }\end{array}$ \\
\hline & & & Multimedia & Multimedia \\
\hline & $\begin{array}{l}\text { System } \\
\text { Quality }\end{array}$ & $\begin{array}{l}\text { Reliability } \\
\text { Usefulness }\end{array}$ & $\begin{array}{l}\text { Reliability } \\
\text { Usefulness }\end{array}$ & $\begin{array}{l}\text { Reliability } \\
\text { Usefulness }\end{array}$ \\
\hline & \multirow{3}{*}{ Usability } & \multirow{3}{*}{ Learnability } & Learnability & Learnability \\
\hline & & & \multirow{2}{*}{ Ease of use } & Ease of use \\
\hline & & & & Ease of acces \\
\hline $\begin{array}{l}\text { Interactio } \\
\mathrm{n} \text { control }\end{array}$ & Task & $\begin{array}{l}\text { Feedback } \\
\text { Action } \\
\text { Expectation }\end{array}$ & $\begin{array}{l}\text { Feedback } \\
\text { Action } \\
\text { Expectation }\end{array}$ & $\begin{array}{l}\text { Feedback } \\
\text { Action } \\
\text { Expectation }\end{array}$ \\
\hline
\end{tabular}


- As pointed by [29], individuals need to awake to determine the processing of information either internally or externally. Thus, Consciousness Value refers to the use of a system of alerting users to something they know or do not know. Some of the responses from the participants are as followed:

"It makes me feel relief as I finally know how to deal with natural disaster (well at least basic knowledge)".

(P3, during-interaction, AP-2)

"I like this application a lot because it helps me manage my weight and exercises."

(P21, post-interaction, AP-1)

- Self- Satisfaction refers to the achievement achieved by using the system. Achievements achieved by the user through the use of the system either through the activities performed or as a result of the activities performed will stimulate the user's emotions. The emotions associated with the achievement of an activity or outcome achievement is defined as the emotion of achievement [30]. Some of the responses from the participants are as followed:

"Able to do something that would improve my health and give a good example to my children."

( $\mathrm{P} 2$, pre-interaction, AP-1)

"It makes me feel happy as it teaches me how to react during a natural disaster"

(P3, during-interaction, AP-2)

Personality refers to attributes that distinguish users from using a system. The unique attributes of these individuals influence the user's emotions towards the system being used. Four aspects referred to Personality in the use of a system which is: Interest, Skill, Knowledge and Relatedness. The following defines the meaning of the four aspects:

- Interest refers to the user's tendency to use the system. The user tends to have a positive attitude towards the system being used and to encourage the user to use the system. Some of the positive responses given by users are as follows:

"This application makes me interested in doing physical activity."

(P13, pre-interaction, AP-1)

"Still interest doing the same every day. Very useful information and update version."

(P8, post-interaction, AP-1)

- Knowledge refers to the knowledge that the user has concerning the system used. For example, if a user does not know the number of calories they eat, it is tough for the user to use applications such as MyFitnessPal that requires users to record the total of the calorie intake of their meals. Here are some responses expressed by participants:
"The calorie input part needed me to estimate the caloric intake for each food that I ate."

(P26, pre-interaction, AP-1)

"Still unable to put in money for house development after spending for defense."

(P18, post-interaction, AP-2)

- Skill refers to the ability of the user to control and use the system. When playing Stop Disaster games, users should think of strategies to prevent flooding in lowlands by applying appropriate development. Some of the responses provided by users are as follows:

"I still unable to finish the game successfully within 10-15 minutes' time frame."

(P23, during-interaction, AP-2)

"All menus easy to be completed."

(P5, during-interaction, AP-1)

- Relatedness refers to the shared goals shared between users and the system. Users to lose or control weight have a trusting relationship with the system used and allow for internalization to occur [31]. Some of the responses that illustrate Relatedness are as followed:

"I always look forward to how to maintain my stamina, and the app is giving a lot of interesting training and good and relevant advice from the trainer."

(P22, pre-interaction, AP-1)

"I feel the system is interesting to me because I want to lose weight..."

(P27, post-interaction, AP-1)

System Control factors consist of four categories: Interface Design, Persuasive Function, Content Presentation, System Quality, and Usability. Interface Design refers to the visual layout of the elements that the user will use to interact with the system. It encompasses two aspects, namely Interface Attractiveness, and Layout. The details of each of these aspects are as follows:

- Interface Attractiveness refers to the use of aesthetic values in system design. For example, the use of dark color and skull images made users feel uncomfortable playing Pandemic 2 game. The example of responses given by the participants was as follows:

"The website has a pleasant look."

(P26, during-interaction, AP-1)

"I regret playing this game plus the color is not interesting."

(P11, during-interaction, AP-2)

- Layout refers to interface layouts that are easy to understand. Unstructured interface sets make it difficult for users to find the information or 
functionality they want, and this will trigger negative emotions in the system being used.

"...selecting the game features has become easier."

(P29, during-interaction, AP-2)

"I hate the background of the game, the layout of the game also quite boring."

\section{(P8, post-interaction, AP-2)}

Persuasive Function refers to the functions provided by the system for users to fulfil their tasks in order to achieve their goals. These functions act as a tool that assists the user to monitor their activities progress using the persuasive applications.

"This application has all the functions that I needed, e.g., calorie counter, BMI calculator."

\section{(P14, pre-interaction, AP-1)}

Content Presentation refers to the approach in the delivery of information used by the system in delivering information to users. This category covers three aspects, namely Multimedia, Information Quality and Data Representation. The descriptions of the three aspects are as follows:

- Multimedia refers to the use of multimedia elements to capture the attention of users. The multimedia aspect is more focused on AP-2 targeting applications, games that use simulation methods as a medium to persuade users of the issues being highlighted.

"It amazed me when the flood occurred; I can see good animation that simulates the flood."

(P16, during-interaction, AP-2)

"It is truly amusing with all graphic impact..."

(P1, post-interaction, AP-2)

- The Information Quality referring to the information provided by the system is complete and accurate.

"Provide knowledge-information in handling flood."

(P19, pre-interaction, AP-2)

"As this app is telling me more about my diets and all details what I have done with me, I am avoiding the things/food not good for me."

(P9, during-interaction, AP-1)

- Data Representation refers to the method of data visualization or data for display to the user.

"The app shows result in a good way (graph)"

(P5, pre-interaction, AP-1)

"The game shows the gradual increase in disease spread starting from green color to red."

(P5, post-interaction, AP-2)

System Quality is a factor that refers to the overall system's behavior to control and to function correctly in terms of Reliability and Usefulness aspects. The details of each of these aspects are as follows:

- Reliability refers to a user's trust towards the system in ensuring that the user can achieve the targeted goal. For examples, the users of Pandemic 2 believed the game in delivering information regarding the diseases spreading. At the same time, through the Fitocracy app, it enables the user to monitor physical activities as one of the ways to lose weight.

"The app enables me to feel good with myself."

(P26, during-interaction, AP-1)

"It provided many exciting experiences playing in trying to achieve a better result."

(P1, post-interaction, AP-2)

- Usefulness refers to the system able to help the user achieved its target goal.

"The game allows me to gain knowledge about preventing and handling flood disaster."

(P19, during-interaction, AP-2)

"I can now practice my favorite exercise with help from this app."

(P17, post-interaction, AP-1)

Usability is a factor in a system that refers to the quality of attributes in assessing how easy for the user to use the system. It encompasses three aspects which were Learnability, Ease of Use and Ease of Access. The details of each of these aspects are as follows:

- Learnability refers to the ability of the system to allow the user to learn how to use it. According to [32], learnability is when the system is easy to learn.

"I thought I had grasped the idea of how to tackle this game and make the virus more lethal, but it was not proven in my previous game. But in this game, there is an improvement, so I think I have some confidence in my strategy now."

(P23, during-interaction, AP-2)

"Having difficulties winning the game even at the easiest level."

(P17, post-interaction, AP-2)

- Ease-of-Use means the system is secure for users to use. This definition is in line with the definition used by [32] and [33] where the system is generally easy to use.

"This app is user-friendly."

(P4, pre-interaction, AP-1)

"It is easy to use this. I love this app."

(P3, during-interaction, AP-1)

- The Ease-of-Access aspect refers to the situation in which the system used is easy for the user to access, for 
example, through websites that can be accessed using computers and mobile phones or through internet access. Overall, the users have made the accessibility aspect as a factor for the post-interaction phase. Here are the responses provided by the participants:

"It is hard to play the app because it requires Internet connection all the time."

\section{(P7, post-interaction, AP-2)}

"I can use the app anywhere as long that I have Internet."

\section{(P11, post-interaction, AP-1)}

Interaction Control factor consists of Tasks category. Tasks refer to the activities that the user is allowed to do while interacting with the system. The three aspects of Tasks are Action, Feedback, and Expectation. The descriptions of these aspects are as follows:

- Actions are selections of action that users can perform to get feedback from the system. Users are given the freedom to make choices in determining the feedback they get from the system. Among the responses expressed by participants were:

"The app can track record from previous days and allows user to edit and re-edit."

\section{(P5, pre-interaction, AP-1)}

"I found many helpful options that maintained my interest."

\section{(P9, post-interaction, AP-1)}

- Feedback is the response and advice given by the system that stimulates emotions and motivates the user. Among the responses expressed by participants about the action:

"Got zero death and zero injuries."

(P1, during-interaction, AP-2)

"I like how the app helps us to monitor our health by showing the graph of food intake for the whole month."

(P11, post-interaction, AP-1)

- Expectation refers to the expectancy that a user can make from using the system, which involves things that users expect the system to do. The following are responses expressed by participants regarding expectations:

"I wish that this game allows for more user control on spreading the diseases."

(P29, pre-interaction, AP-2)

"The app cannot give a prediction of weight after listing all the foods that the user had eaten."

(P5, post-interaction, AP-1)

\section{DISCUSSIONS}

\section{A. Emotional States}

This study reveals that, in the pre-interaction stage, most users experience the feeling of "interest", "pleasure", "amusement", "love," and "joy". All of these emotions are high-control positive emotions except for "love," which are low-control positive emotions. Responses from the users towards the AP-1 and AP-2 has always related to the aspect of Interest, the tendency of using the system, making many of the users choosing "interest" and "pleasure" emotion as the emotion that they experienced since interest is the emotion that is activated when one experiences engagement, interest, and curiosity [34]. The findings suggest that people decide to adopt a system when they feel pleasant or having positive thought about the system.

The study found that, in during interaction stage, users experience a variety of emotions mostly the feeling of "interest", “contentment", "relief", "disappointment", "joy", "pleasure", and "guilt". These emotions are contained in highcontrol positive emotion (i.e., interest, pleasure, joy), lowcontrol positive emotion (i.e., relief, contentment) and lowcontrol negative emotion (i.e. disappointment, guilt). Findings from the study suggest that System Quality, Content Presentation, Interface Design, Personality, Individual Awareness and Task are the elements that triggered those emotions.

For the post-interaction stage, the study reveals that users experience more emotions of "interest", "love", "pleasure", "disappointment" and "hate". Those triggered emotions are a combination of four groups, which are high-control positive emotion (i.e., interest, pleasure), low-control positive emotion (i.e., love), low-control negative emotions group (i.e., disappointment) and high-control negative emotion group (i.e., hate). The findings suggest that all eight elements affect users' emotions at this particular stage. Overall, the triggered emotions for the three interaction stages are dominated by emotions from a high-control positive emotions group indicating that the persuasive applications used in the experiment triggered positive emotions among the users in the persuasion process. These findings agreed with previous studies by [35] and [36] that persuasion is perceived when the user felt positive emotions.

\section{B. Stimulation Factors of Emotions}

In using both types of persuasive applications, it is found that the emotions experienced by the user are under the control of all three factors; User, System and Interaction which encompasses eight elements namely Individual Awareness, Personality, Interface Design, Persuasive Function, Content Presentation, System Quality, Usability, and Tasks. However, what distinguishes these two types of persuasive applications are the aspects that stimulate user emotion found through the open code.

For AP-1 persuasive applications, the pre-interaction stage shows 18 aspects that dominate the user's emotions except for Multimedia, Ease of Use and Ease of Access elements. However, in the during-interaction stage, aspects such as Skills, Layout, Data Representation, Multimedia, Learnability, 
and Ease of Access are not affected by the emotions of the user, and this results in only 14 aspects of the user's emotions. However, in the post-interaction stage, 17 aspects succeeded in affecting the user's emotions except for aspects such as Skills, Layout, and Multimedia. Overall, the multimedia aspect was not seen as a contributing factor to the emotion experienced by users in the use of persuasive applications of tools.

For AP-2 persuasive applications, 12 aspects were found to affect the user's emotions in the pre-interaction stage except for aspects such as Layout, Data Representation, Multimedia, Reliability, Ease of Use, Ease of Access, as well as two aspects of Individual Awareness that include Consciousness Value and Self-Satisfaction. However, in the duringinteraction stage found 17 elements that impacted the user's emotions except for aspects such as Persuasive Function, Ease of Use, and Ease of Access. The post-interaction stage also found 17 aspects that influenced the user's emotions except for aspects such as Skills, Learnability, and Ease of Use. Overall, it was found that the Ease of Use aspect was not a contributing factor to the emotions that users experienced in using persuasive applications that fall under the medium category. This finding also found that Ease-of-Access aspect was only evaluated and considered relevant by users in the use of both types of persuasive applications in the post-interaction stage only.

\section{Relation between the Emotional States and Stimulation Factors}

The findings show that "interest", "pleasure", and "joy" are the positive emotions that dominate users at each interaction stage. At the same time, "disappointment" is the negative emotions that always triggered in users across the interaction stages. The negative emotions that users felt were caused by the aspects of Task, Individual Awareness, Personality and Interface Design that cover all three factors; User Control, System Control and Interaction Control. The comparison with the outcomes of previously user emotion study [25] reveals a similar pattern in the emotions that users experience even by assessing the different types of stimuli, which in this case a higher learning institution website designed using standard Kansei-based guidelines. However, the study did not identify what specifically trigger those positive emotions. Although the emotion of "interest" in the present study receives the highest values in every interaction stages, this does not mean that the same thing will happen whenever emotions are directed to a tool or device; nevertheless, this offers a test of the significance of these measures to design-related research. The emotion of "interest" is usually correlated with innovative practices, the growth of skills and knowledge, the learning of new abilities, and consistency in an effort [34]. While most previous studies [22] [23] [25] successfully evaluates user emotions to address the user response towards the design of technology, this study, at the same time, identifies the factors and elements that are addressing those user emotions. The findings show that the highlighted elements are the potential antecedents that affect the trust of the user, hence becoming a strategy that needs to be addressed for constructing an emotion-based trust design framework for persuasive technology.

\section{CONCLUSION}

This study has contributed to the findings on what exactly users feel when using a persuasive application and the factors that triggered the experienced emotions to the body of knowledge of Persuasive Technology field interest. User emotions in each interaction stage can vary because of different factors such as User, System and Interaction and the elements of the factor, for examples Individual Awareness, Personality, Interface Design, Persuasive Function, Content Presentation, System Quality, Usability, and Tasks. The findings can be used to construct a design framework of persuasive technology that can bring an emotional impact to the user by focusing on the factors with appropriate design principles or strategies. The designing framework, however, will be reported in the other paper, after triangulation with diary studies' results.

\section{ACKNOWLEDGMENT}

Thank you to the Malaysian government for granting funds on this work, which is part of the research under the Fundamental Research Grant Scheme (FRGS) (FRGS/2/2014/ICT01/UKM/02/3) by the Ministry of Education.

\section{REFERENCES}

[1] B. J. Fogg, Persuasive Technology Using Computers to Change What We Think and Do. Morgan Kaufmann: San Francisco, 2003.

[2] H. Oinas-Kukkonen and M. Harjuuma, "Persuasive systems design: key issues, process model, and system features," Communications of the Association for Information Systems, vol. 24, no. 28, pp. 485-500, Mar. 2009.

[3] J. Hamari, J. Koivisto, T. Pakkanen, "Do persuasive technologies persuade? A review of empirical studies," in Persuasive Technology. PERSUASIVE 2014. Lecture Notes in Computer Science, vol. 8462, A. Spagnolli, L. Chittaro, and L. Gamberini, Eds. Cham, Springer, 2014, pp. 118-136.

[4] N. Mohamad Ali., S. Z Abdullah, J. Salim, R. Sulaiman, H. B. Zaman and H. Lee, "Exploring user experience in game using heart rate device," Asia-Pacific Journal of Information technology and Multimedia, vol.1, no. 2, pp. 28-36, 2012.

[5] H. Petrie and J, Precious, "Measuring user experience of websites: think aloud protocols and an emotion word prompt list," CHI 2010, pp. 36733678, April 2010.

[6] F. Zhou, X. Qu, M. G. Helander and J. Jiao, "Affect prediction from physiological measures via visual stimuli," International Journal of Human-Computer Studies, vol. 69, no. 12, pp. 801-819, 2011.

[7] I. Lopatovska, and I. Arapakis, "Theories, methods and current research on emotions in library and information science, information retrieval and human-computer interaction," Information Processing and Management, vol. 47, no. 4, pp. 575-592, 2011.

[8] R. E. Petty and P. Brinol, "Emotion and persuasion: Cognitive and metacognitive processes impact attitudes," Cognition and Emotion, vol. 29, no. 1, pp. 1-26, 2014.

[9] J. Forlizzi and K. Battarbee, "Understanding experience in interactive systems understanding experience in interactive systems," 5th Conference on Designing interactive systems 2004, pp. 261-268, August 2004.

[10] D. Lottridge, M. Chignell and A. Jovicic, "Affective interaction: Understanding, evaluating, and designing for human emotion," Reviews of Human Factors and Ergonomics, vol. 7, no. 1, pp. 197-217, 2011.

[11] K. R. Scherer, "What are emotions? And how can they be measured?" Social Science Information, vol. 44, no. 4, pp 695-729, 2005.

[12] T. Partala, "Affective information in Human-Computer Interaction," Ph.D. dissertation, Department of Computer Sciences, University of Tampere, Finland, 2005. 
[13] K. Boehner, R. Depaula, P. Dourish, P. Sengers, A. C. Zaidan and U.C. Irvine, "How emotion is made and measured," International Journal of Human-Computer Studies, vol.65, pp. 275-291, 2007.

[14] P. Ekman and W. V. Friesen, Unmasking the face. A guide to recognizing emotions from facial clues., Prentice-Hall: Englewood Cliffs, New Jersey, 1975.

[15] C. Darwin, The expression of the emotions in man and animals, Cambridge University Press, 2009.

[16] I. Hupont, S. Baldassarri and E. Cerezo, "Facial emotional classification: from a discrete perspective to a continuous emotional space," Patter Analysis \& Applications, vol. 16, no. 1, pp. 41-54, 2013.

[17] E. Krahmer, J. V. Dorst and N. Ummelen, "Mood, persuasion and information presentation. The influence of mood on the effectiveness of persuasive digital documents," Information Design Journal, vol. 12, no. 3, pp. 40-52, 2004.

[18] E. Schaffer, "PET Research: Looking Deeper to Understand Motivations," 2010. https://humanfactors.com/downloads/whitepapers/ PET-research.pdf.

[19] B. H. Marcus and L. H. Forsyth, Motivating People to be Physically Active: Physical activity intervention series, Human Kinetic, Champaign: IL, 2003.

[20] W. N. Wan Ahmad, N. Mohamad Ali, "The impact of persuasive technology on user emotional experience and user experience over time," Journal of Information Communication Technology, vol. 17, no. 4, pp. 601-628, 2018.

[21] K. R. Scherer, V. Shuman, J. R. J. Fontaine and C. Soriano, "The GRID meets the wheel: Assessing emotional feeling via self-report," in Components of emotional meaning: A sourcebook, J. R. J. Fontaine, K. R. Scherer, and C. Soriano, Eds. Oxford: Oxford University Press, 2013, pp. 281-298.

[22] J. M. dos. Santos, "Gaia: intelligent control of virtual environments," $\mathrm{PhD}$ dissertation, Universidade Tecnica de Lisboa, 2008. https://dspace.ist.utl.pt/bitstream/2295/236570/1/Dissertacao da Tese de Mest\%0Arado - FINAL - Revised - Jorge Santos.pdf.

[23] M. T. Longhi, D. F. Pereira, M. Bercht, \& P. A. Behar, "An experiment to understand how the affective aspects can be detected in virtual learning environments," CINTED-UFRGS, vol.7, 2009.

[24] G. F. G. Laurans, "On the moment-to-moment measurement of emotion during person-product interaction," Technische University of Delft, 2011.
[25] P. Turumugon, A. Baharum, N. H. Nazlan, N. A. M. Noh, N. A. M. Noor and E. A. Rahim, "Users' emotional evaluation towards kanseibased higher learning institution website using Geneva Emotion Wheel," Indonesian Journal of Electrical Engineering and Computer Science, vol. 16 , no. 3, pp. 1547-1554, 2019.

[26] D. Silverman, Interpreting Qualitative Data., London: Sage Publications Ltd., 2014.

[27] J. R. Dunn and M. E. Schweitzer, "Feeling and believing: the influence of emotion on trust," Journal of Personality and Social Psychology, vol. 88, no. 5, pp. 736-748, 2005.

[28] J. So, C. Achar, D. Han, N. Agrawal, A. Duhachek and D. Maheswaran, "The psychology of appraisal: Specific emotions and decision-making," Journal of Consumer Psychology, vol. 25, no. 3, pp. 359-371, 2015.

[29] A. Morin, "Levels of consciousness and self-awareness: A comparison and integration of various neurocognitive views," Consciousness and Cognition, vol. 15, pp. 358-371, 2006.

[30] R. Pekrun, A.J., Elliot. And M.A., Maier, "Achievement goals and discrete achievement emotions: A theoretical model and prospective test," Journal of Education Psychology, vol. 98, pp. 583-597, 2006.

[31] R. M. Ryan, H. Patrick, E. L. Deci and G. C. Williams, "Facilitating health behaviour change and its maintenance: interventions based on Self-Determination Theory," The European Health Psychologist, vol. 10, pp. 2-5, 2008.

[32] J. Xu, K. Le, A. Deitermann and E. Montague, "How different types of users develop trust in technology: A qualitative analysis of the antecedents of active and passive user trust in a shared technology," Applied Ergonomics, vol. 45, pp. 1495-1503, 2014.

[33] Z. Yan, R. Kantola and P. Zhang, "A research model for humancomputer trust interaction," in 2011I EEE 10th International Conference on Trust, Security and Privacy in Computing and Communications, pp. 274-281, November 2011.

[34] C. E. Izard, The Psychology of Emotions. New York: Plenum Press, 1991.

[35] V. Griskevicius, M. N. Shiota and S. L. Neufeld, "Influence of different positive emotions on persuasion processing: a functional evolutionary approach," Emotion, vol. 10, no. 2, pp. 190-206, 2010.

[36] J. V. Riet, R. A. C. Ruiter, M. Q. Werrij, M. J. J. M. Candel and H. de Vries, "Distinct pathways to persuasion: The role of affect in message - framing effects," European Journal of Social Psychology, vol. 40, no. 7, pp. 1261-1276, 2010. 